\title{
Predictors of immunotherapy-induced immune-related adverse events
}

\author{
A. Kartolo MD, ${ }^{*}$ J. Sattar MSc, ${ }^{*}$ V. Sahai $\mathrm{MSc}^{+}$T. Baetz MD, ${ }^{* \neq}$ and J.M. Lakoff $\mathrm{MD}^{*+\S}$
}

\section{ABSTRACT}

Purpose We aimed to elucidate predictive factors for the development of immune-related adverse events (iraes) in patients receiving immunotherapies for the management of advanced solid cancers.

Methods This retrospective study involved all patients with histologically confirmed metastatic or inoperable melanoma, non-small-cell lung cancer, or renal cell carcinoma receiving immunotherapy at the Cancer Centre of Southeastern Ontario. The type and severity of irAEs, as well as potential protective and exacerbating factors, were collected from patient charts.

Results The study included 78 patients receiving ipilimumab (32\%), nivolumab (33\%), or pembrolizumab (35\%). Melanoma, non-small-cell lung cancer, and renal cell carcinoma accounted for $70 \%, 22 \%$, and $8 \%$ of the cancers in the study population. In 41 patients (53\%) iraes developed, with multiple iraes developing in 12 patients (15\%). In most patients $(70 \%)$, the iraEs were of severity grade 1 or 2 . Female sex [adjusted odds ratio $\left(\mathrm{OR}_{\text {adj }}\right): 0.094 ; 95 \%$ confidence interval (CI): 0.021 to $0.415 ; p=0.002$ ] and corticosteroid use before immunotherapy (OR adj: $_{0} 0.143 ; 95 \%$ Сг: 0.036 to $0.562 ; p=0.005$ ) were found to be associated with a protective effect against iraEs. In contrast, a history of autoimmune disease ( $\mathrm{OR}_{\text {ad: }}: 9.55 ; 95 \%$ CI: 1.34 to $\left.68.22 ; p=0.025\right)$, use of CTLA-4 inhibitors ( $\mathrm{OR}_{\text {adj }}: 6.25 ; 95 \%$ Cr: 1.61 to $24.25 ; p=0.008)$, and poor kidney function of grade 3 or greater ( $\mathrm{OR}_{\mathrm{adj}}: 10.66 ; 95 \% \mathrm{CI}: 2.41$ to $\left.47.12 ; p=0.025\right)$ were associated with a higher risk of developing iraEs. A Hosmer-Lemeshow goodness-of-fit test demonstrated that the logistic regression model was effective at predicting the development of irAEs (chi-square: 1.596; $d f=7 ; p=0.979$ ).

Conclusions Our study highlights several factors that affect the development of iraes in patients receiving immunotherapy. Although future studies are needed to validate the resulting model, findings from the study can help to guide risk stratification, monitoring, and management of irAEs in patients given immunotherapy for advanced cancer.

Key Words Predictors, immunotherapy, immune-related adverse events, advanced solid cancers

\section{INTRODUCTION}

Immunomodulation has recently become an important and promising line of therapy for the treatment of advanced melanoma and an increasing number of other cancer types $^{1}$. This new line of immunotherapies consists of antibodies that exert their effect by targeting the immune checkpoint inhibitors. Currently available therapy consists of inhibitors of CTLA-4, PD-1, and PD-L1. The three most common immunotherapies are ipilimumab (CTLA-4 inhibitor), nivolumab (PD-1 inhibitor), and pembrolizumab (PD-1 inhibitor).
In a landmark clinical trial by Hodi et al. ${ }^{2}$, administration of ipilimumab in patients with stage III or IV melanoma was shown to significantly increase median survival. Similarly, pembrolizumab and nivolumab have both been shown to improve overall survival and progression-free survival in patients with metastatic melanoma ${ }^{3,4}$. In addition, compared with ipilimumab, the PD-1 inhibitors have demonstrated better efficacy with fewer adverse events, leading to approval by Health Canada for their use in ipilimumab-resistant advanced melanoma in $2014^{5}$ and in ipilimumab-naïve patients in 2015. The PD-1 inhibitors were subsequently shown to improve survival in patients

Correspondence to: Joshua Matthew Lakoff, Faculty of Health Sciences, Queens' University, Sydenham 3, Room 013-2, Hotel Dieu Hospital, 166 Brock Street, 
with advanced non-small-cell lung cancer ${ }^{6}$ and renal cell carcinoma ${ }^{7}$. Since then, immune checkpoint inhibitors have been used in the treatment of various other advanced cancers, including urothelial cancer, head-and-neck carcinoma, hepatocellular carcinoma, and small-cell lung cancer.

Despite the clinical efficacy of immune checkpoint inhibitors, a growing body of evidence has shown that various immune-related adverse events (irAEs) occur in a significant portion of patients treated with both antiCTLA-4 and anti-PD-1 antibodies ${ }^{1}$. Most iraEs, including diarrhea and nausea, tend to be mild and self-limiting, but in fewer than $10 \%$ of patients treated with immune checkpoint inhibitors, severe (grade 3 or 4), potentially life-threatening irAes can occur ${ }^{8}$. The iraEs can be classified based on the physiologic system affected, the most commonly affected being the endocrine, dermatologic, gastrointestinal, and respiratory systems ${ }^{9}$. Although irAEs can occasionally be managed with a brief course of corticosteroid therapy, grade 3 or 4 iraes typically require termination of immunotherapy and long-term treatment with steroids or other immunosuppressive medications such as infliximab.

With the increasing use of immunotherapy in the management of cancers, determining risk factors for the development of irAEs attributable to this group of drugs can provide invaluable help for predicting and better managing patients who develop irAEs. Some of the hypothesized risk factors include low muscle attenuation ${ }^{10}$, history of autoimmune disease ${ }^{9}$, chronic infections such as HIV and hepatitis $^{11-13}$, and use of medications with autoimmune side effects ${ }^{14}$. However, to the best of our knowledge, no studies have effectively validated those potential risk factors. Several studies have investigated biomarkers that might be predictive of an increased occurrence of irAEs, including increased T-cell repertoire ${ }^{15}$, eosinophil count ${ }^{16}$, gene expression of CD177 and CEACAM1 ${ }^{17}$, and blood levels of interleukin $7^{18}$. Nevertheless, important knowledge gaps remain for the effective identification of patients receiving immune checkpoint inhibitors who are at risk for developing iraEs. In the present retrospective cohort study, we aimed to comprehensively analyze patient factors associated with irAEs and to develop a predictive tool for optimal clinical decision-making and patient management.

\section{METHODS}

All patients with histologically confirmed metastatic melanoma, non-small-cell lung cancer, or renal cell carcinoma who were managed at the Cancer Centre of Southeastern Ontario (a tertiary care cancer centre) and who received ipilimumab, pembrolizumab, or nivolumab between 1 January 2012 and 18 April 2017 were included in the study. Eligible patients $(n=89)$ were identified by searching a computerized pharmacy order-entry database. Patients were excluded if they were enrolled in a clinical trial $(n=10)$. A patient with hepatocellular carcinoma was also excluded because of the small sample size, leaving 78 patients for the study analysis. The study was approved by the Queen's University Health Sciences and Affiliated Teaching Hospitals Research Ethics Board.
All data were obtained from patient charts. Potential risk factors were categorized into two groups: exacerbating factors and protective factors. A literature review was conducted to support the inclusion of potential risk factors. Exacerbating factors were defined as contributors that might lead to immune dysfunction and a potentially increased risk of irAEs. Those factors included a history of autoimmune disease ${ }^{9}$, history of chronic infection (HIV, hepatitis, shingles) ${ }^{11-13}$, allergies (medication or environmental) ${ }^{19}$, previous iraEs, high body mass index ${ }^{20}$, impaired kidney function ${ }^{21,22}$, or specific medications ${ }^{14}$ such as antiarrhythmics, antihypertensives, antipsychotics, anticonvulsants, and statins. Protective factors included medications with immunosuppressive mechanisms ${ }^{14}$ steroids, allopurinol, nonsteroidal anti-inflammatory drugs, salicylates, and metformin - that might lead to a lower rate of irAEs. All medications identified were in use before the start of immunotherapy.

The iraEs were collected as defined in previous studies $^{9,11,12,23}$. Common side effects identified included skin toxicity, gastrointestinal toxicity, and endocrinopathy. Skin toxicity was defined as development of a maculopapular rash or vitiligo. Gastrointestinal toxicity was defined as having watery bowel movements in the absence of an infectious cause or as colitis confirmed by endoscopy. Endocrinopathy included hypophysitis, thyroiditis, adrenal insufficiency, and diabetic ketoacidosis. Because immunotherapy has the potential to affect any organ system, an "other" category was used to collect instances of uncommon iraEs. The toxicity severity was graded from 1 to 5 according to the Common Terminology Criteria for Adverse Events, version $4.0^{24}$. The primary outcome was defined as the presence of an iraE. Secondary outcomes included multiple irAEs ( 2 or more) and an irAE severity of grade 3 or greater.

\section{Statistical Analysis}

All statistical analyses were conducted using the IBM SPSS Statistics software application (version 24.0: IBM, Armonk, NY, U.S.A.) for Windows (Microsoft Corporation, Redmond, WA, U.S.A.). Descriptive statistics provide an overview of the characteristics of the study population. Bivariate analyses assessed the relationship between potential predictors and irAE incidence rates. Results are reported as odd ratios (oRs) and means with 95\% confidence intervals (CIS). Statistical significance was accepted at $p<0.05$. A logistic regression model was then used to determine the association between irAEs and the significant predictors identified in the bivariate analyses. To account for the sample size when deriving the model, all variables significant at the alpha level of 0.1 were entered into the multiple logistic regression model-but only if that factor was present in at least $5 \%$ of patients with that toxicity event. The backward stepwise elimination method, based on maximum partial likelihood estimates, was used to develop a parsimonious set of predictors while maintaining biologic integrity. The Wald statistic was used to determine the significances of the regression coefficients, with the alpha level set at 0.05 . The integrity and predictive accuracy of the model were assessed using the Hosmer-Lemeshow goodness-of-fit test and a receiver operating characteristic curve respectively. 
PREDICTORS OF IMMUNOTHERAPY-INDUCED IMMUNE-RELATED ADVERSE EVENTS, Kartolo et al.

\section{RESULTS}

The 78 study patients (30 women, 48 men) had an average age of 66 years (range: $23-85$ years). Despite the 23-yearolds $(n=2)$ being outliers, all patients were included in the analysis given the relevance of a broad age range to a general cancer centre practice. Melanoma, non-smallcell lung cancer, and renal cell carcinoma accounted for, respectively, $70 \%, 22 \%$, and $8 \%$ of the cancers in the study population. The distribution of nivolumab (33\%), pembrolizumab (35\%), and ipilimumab (32\%) was similar in the study population. Approximately half the patients in the study population (53\%) developed an iraE, and $15 \%$ developed multiple irAEs. Most patients (70\%) developed an irAE of grade 1 or 2 . The irAes most commonly involved the skin $(22 \%)$, gastrointestinal system (17\%), and endocrine system (14\%). Table I summarizes baseline patient health information, treatment modality, and irae types.

Table II presents the results of the bivariate analysis of potential predictors and irAEs. After adjusting for confounding factors, female sex [adjusted oR $\left(\mathrm{OR}_{\mathrm{adj}}\right): 0.094 ; 95 \%$ CI: 0.021 to $0.415 ; p=0.002$ ] and use of corticosteroids before immunotherapy (oR ${ }_{\text {adj }}$ : $0.143 ; 95 \%$ CI: 0.036 to $0.562 ; p=$ 0.005 ) were found to be associated with a protective effect against the development of irAEs. Conversely, a history of autoimmune disease $\left(\mathrm{OR}_{\mathrm{adj}}\right.$ : 9.55; 95\% CI: 1.34 to $68.22 ; p=$ 0.025 ), use of CTLA- 4 inhibitors ( $\mathrm{OR}_{\text {adj }}: 6.25 ; 95 \% \mathrm{CI}: 1.61$ to $24.25 ; p=0.008$ ), and poor kidney function of grade 3 and greater (oR ${ }_{\text {adj }}$ : 10.66; 95\% CI: 2.41 to $\left.47.12 ; p=0.025\right)$ were found to be associated with a higher risk of developing irAEs. No significant associations between iraEs and age, body mass index, number of distant metastatic sites, smoking history, and allergy history were found. Analysis of risk for patients who had already experienced an irAe and who were subsequently treated with another immunotherapy was not possible because of the small sample size $(n=11)$. Table III presents details.

Patients who used statin medications, who had radiation therapy during immunotherapy, or who had melanoma showed an increased risk for irAEs on bivariate analysis. However, in the subsequent logistic regression modelling, those factors were not found to be independent predictors. The Hosmer-Lemeshow test showed that, overall, the model was excellent at predicting which patient would or would not develop an irae (chi-square: 1.596; $d f=7 ; p=0.979$ ). The model was shown to have good predictive accuracy as assessed by the area under the receiver operating characteristic curve. The area under the curve was $82.4 \%(95 \%$ CI: $73.4 \%$ to $91.4 \%$ ), and it was found to be significantly different relative to the reference line (Figure 1).

\section{DISCUSSION}

To the best of our knowledge, the present study is the first to evaluate factors that predict risk for irAEs developing in patients receiving PD-1 and CTLA-4 inhibitors. Although irAEs were common in our study (more than $50 \%$ incidence rate), most patients (70\%) developed iraEs of mild-tomoderate severity, and only a small proportion (15\%) developed multiple iraEs. After adjusting for confounding factors, our study suggests that steroid use before
TABLE I Demographics and health parameters for the 78 study patients

\begin{tabular}{|c|c|}
\hline & Value \\
\hline \multicolumn{2}{|l|}{$\operatorname{Sex}[n(\%)]$} \\
\hline Men & $48(62)$ \\
\hline Women & $30(38)$ \\
\hline \multicolumn{2}{|l|}{ Age (years) } \\
\hline Mean & 66.2 \\
\hline Range & $23-85$ \\
\hline \multicolumn{2}{|c|}{ Body mass index } \\
\hline Mean & 27.6 \\
\hline Range & $16.7-45.8$ \\
\hline \multicolumn{2}{|c|}{ Cancer type $[n(\%)]$} \\
\hline Melanoma & $55(70)$ \\
\hline NSCLC & $17(22)$ \\
\hline RCC & $6(8)$ \\
\hline
\end{tabular}

Site or sites distant metastasis ( $n$ )

$\begin{array}{lc}\text { Mean } & 2.35 \\ \text { Range } & 0-7\end{array}$

Past medical history $[n(\%)]$

Autoimmune disease

$12(15)$

Chronic infection

$5(6)$

History of allergies [n (\%)]

Medication

$20(26)$

Environmental substances

$6(7)$

Medication use [ $n(\%)]$

Anti-arrhythmics

$20(26)$

Antihypertensives

37 (47)

Antipsychotics

3 (4)

Anticonvulsants

$5(6)$

Statins

$32(41)$

Steroids

24 (31)

Allopurinol

2 (3)

NSAIDs

9 (12)

Salicylates

10 (13)

Metformin

9 (12)

Baseline eGFR ${ }^{\mathrm{a}}[n(\%)]$

Grade 1

25 (32)

Grade 2

38 (49)

Grade 3a

Grade 3b

10 (13)

Grade 4

1 (1)

Immunotherapy agent [ $n(\%)]$

Ipilimumab

25 (32)

Pembrolizumab

27 (35)

Nivolumab

26 (33)

Overlap of RT and immunotherapy [ $n(\%)]$

Yes

37 (47)

No

41 (53) 
TABLE I Continued

\begin{tabular}{lc}
\multicolumn{1}{c}{ Variable } & Value \\
\hline $\begin{array}{l}\text { Immune-related AEs }[n(\%)] \\
\text { Yes, at least } 1\end{array}$ & $41(53)$ \\
No & $37(47)$ \\
Yes, multiple & $12(15)$ \\
No, multiple & $66(85)$ \\
Yes, $\geq$ grade 3 & $12(30)$ \\
No, $\geq$ grade 3 & $28(70)$ \\
System affected by immune-related AEs [n (\%)] & \\
Skin & $17(22)$ \\
Gastrointestinal & $13(17)$ \\
Endocrine & $11(14)$ \\
Other & $13(17)$ \\
\hline
\end{tabular}

a As defined by The Renal Association (Bristol, U.K.): grade 1, $\geq 90 \mathrm{~mL} / \mathrm{min} / 1.73 \mathrm{~m}^{2}$; grade $2,60-89.9 \mathrm{~mL} / \mathrm{min} / 1.73 \mathrm{~m}^{2}$; grade $3 \mathrm{a}$, $45-59.9 \mathrm{~mL} / \mathrm{min} / 1.73 \mathrm{~m}^{2}$; grade $3 \mathrm{~b}, 30-44.9 \mathrm{~mL} / \mathrm{min} / 1.73 \mathrm{~m}^{2}$; grade $4,15-29.9 \mathrm{~mL} / \mathrm{min} / 1.73 \mathrm{~m}^{2}$.

b Respiratory $(n=5)$, renal $(n=3)$, musculoskeletal $(n=4)$, neurologic $(n=1)$.

NSCLC $=$ non-small-cell lung cancer; $\mathrm{RCC}=$ renal cell carcinoma; NSAIDs = nonsteroidal anti-inflammatory drugs; eGFR - estimated glomerular filtration rate; $\mathrm{RT}=$ radiotherapy; $\mathrm{AEs}=$ adverse events. immunotherapy, sex, a history of autoimmune disease, immunotherapy with a cTLA- 4 monoclonal antibody, and abnormal kidney function have statistically significant associations with irAE incidence rates.

Corticosteroid use before initiation of immunotherapy (33\%, $n=26)$ was associated with a lower incidence of irAEs. The most common indications for steroid treatment were post-radiation management $(54 \%, n=14)$ and symptomatic brain metastases $(27 \%, n=7)$. The average dose and duration of corticosteroid before the start of immunotherapy was $35.2 \pm 2.49 \mathrm{mg}$ (prednisone equivalent) and $4.9 \pm 5.5$ weeks respectively. Several previous studies have analyzed the effect of immunosuppressive therapy (indicated for irAE management) on immunotherapy efficacy. In a pooled analysis of four clinical trials of nivolumab monotherapy for patients with advanced melanoma, immunosuppressive therapy as indicated for iraEs was not associated with differences in the immunotherapy response rate ${ }^{25}$. Another study also showed no differences in the efficacy of ipilimumab (response rate and overall survival) with immunosuppressive therapy in the context of a nonclinical trial in advanced melanoma patients ${ }^{26}$. Future studies are required to evaluate the safety and efficacy of steroids given concurrently with immunotherapy, especially in patients who are receiving a significant dose of steroids before they start treatment, because those patients were excluded from clinical trials.

TABLE II Bivariate analyses of risk factors in relation to immune-related adverse events

\begin{tabular}{|c|c|c|c|c|c|}
\hline \multirow[t]{2}{*}{ Factor } & \multicolumn{4}{|c|}{ Immune-related adverse events } & \multirow{2}{*}{$\underset{\text { Value }^{\mathrm{a}}}{\boldsymbol{p}}$} \\
\hline & Yes & No & OR & $95 \% \mathrm{CI}$ & \\
\hline \multicolumn{6}{|l|}{$\operatorname{Sex}[n(\%)]$} \\
\hline Men & $30(63)$ & $18(38)$ & 0.35 & 0.14 to 0.89 & 0.036 \\
\hline Women & $11(37)$ & $19(63)$ & & & \\
\hline Mean age (years) & $67.0 \pm 13.2$ & $65.3 \pm 11.8$ & -0.01 (mean diff.) & -7.00 to 3.72 & 0.57 \\
\hline Mean body mass index & $28.3 \pm 5.5$ & $26.9 \pm 5.5$ & 0.022 (mean diff.) & -3.74 to 0.85 & 0.22 \\
\hline \multicolumn{6}{|l|}{ Cancer type $[n(\%)]$} \\
\hline Melanoma & $34(62)$ & $21(38)$ & NA & & 0.040 \\
\hline NSCLC & $5(29)$ & $12(71)$ & & & \\
\hline $\mathrm{RCC}$ & $2(33)$ & $4(67)$ & & & \\
\hline Mean site or sites distant metastasis ( $n$ ) & $2.4 \pm 1.4$ & $2.3 \pm 1.5$ & -0.093 & -0.739 to 0.553 & 0.775 \\
\hline \multicolumn{6}{|l|}{ Past medical history $[n(\%)]$} \\
\hline \multicolumn{6}{|l|}{ Autoimmune disease } \\
\hline Yes & $8(67)$ & $4(33)$ & 2.00 & 0.55 to 7.3 & 0.36 \\
\hline No & $33(50)$ & $33(50)$ & & & \\
\hline \multicolumn{6}{|l|}{ Chronic infection } \\
\hline Yes & $2(40)$ & $3(60)$ & 0.58 & 0.09 to 3.69 & 0.66 \\
\hline No & $39(53)$ & $34(47)$ & & & \\
\hline \multicolumn{6}{|l|}{ History of allergies $[n(\%)]$} \\
\hline \multicolumn{6}{|l|}{ Medication } \\
\hline Yes & $12(60)$ & $8(40)$ & 1.50 & 0.53 to 4.21 & 0.60 \\
\hline No & $29(50)$ & $29(50)$ & & & \\
\hline
\end{tabular}


PREDICTORS OF IMMUNOTHERAPY-INDUCED IMMUNE-RELATED ADVERSE EVENTS, Kartolo et al.

TABLE II Continued

\begin{tabular}{|c|c|c|c|c|c|}
\hline \multirow[t]{2}{*}{ Factor } & \multicolumn{4}{|c|}{ Immune-related adverse events } & \multirow{2}{*}{$\underset{\text { Value }^{\mathrm{a}}}{p}$} \\
\hline & Yes & No & OR & $95 \% \mathrm{Cl}$ & \\
\hline \multicolumn{6}{|c|}{ History of allergies $[n(\%)]$ continued } \\
\hline \multicolumn{6}{|l|}{ Environmental substances } \\
\hline Yes & $4(67)$ & $2(33)$ & 1.89 & 0.33 to 11.0 & 0.68 \\
\hline No & $37(51)$ & $35(49)$ & & & \\
\hline \multicolumn{6}{|l|}{ Medication use [ $n(\%)]$} \\
\hline \multicolumn{6}{|l|}{ Anti-arrhythmics } \\
\hline Yes & $13(65)$ & $7(35)$ & 1.99 & 0.69 to 5.71 & 0.30 \\
\hline No & $28(48)$ & $30(52)$ & & & \\
\hline \multicolumn{6}{|l|}{ Antihypertensives } \\
\hline Yes & $21(57)$ & $16(43)$ & 1.38 & 0.56 to 3.37 & 0.51 \\
\hline No & $20(49)$ & $21(51)$ & & & \\
\hline \multicolumn{6}{|l|}{ Antipsychotics } \\
\hline Yes & $2(67)$ & $1(33)$ & 1.85 & 0.16 to 21.2 & 1.00 \\
\hline No & $39(52)$ & $36(48)$ & & & \\
\hline \multicolumn{6}{|l|}{ Anticonvulsants } \\
\hline Yes & $2(40)$ & $3(60)$ & 0.58 & 0.09 to 3.69 & 0.66 \\
\hline No & $39(53)$ & $34(47)$ & & & \\
\hline \multicolumn{6}{|l|}{ Statins } \\
\hline Yes & $22(69)$ & $10(31)$ & 3.13 & 1.21 to 8.09 & 0.022 \\
\hline No & $19(41)$ & $27(59)$ & & & \\
\hline \multicolumn{6}{|l|}{ Steroids } \\
\hline Yes & $7(29)$ & $17(71)$ & 0.24 & 0.09 to 0.69 & 0.007 \\
\hline No & $34(63)$ & $20(37)$ & & & \\
\hline \multicolumn{6}{|l|}{ Allopurinol } \\
\hline Yes & $2(100)$ & $0(0)$ & NA & & 0.50 \\
\hline No & $39(51)$ & 37 (49) & & & \\
\hline \multicolumn{6}{|l|}{ NSAIDs } \\
\hline Yes & $5(56)$ & $4(44)$ & 1.15 & 0.28 to 4.63 & 1.00 \\
\hline No & $36(52)$ & $33(48)$ & & & \\
\hline \multicolumn{6}{|l|}{ Salicylates } \\
\hline Yes & $7(70)$ & $3(30)$ & 2.33 & 0.56 to 9.79 & 0.32 \\
\hline No & $34(50)$ & $34(50)$ & & & \\
\hline \multicolumn{6}{|l|}{ Metformin } \\
\hline Yes & $5(56)$ & $4(44)$ & 1.15 & 0.28 to 4.63 & 1.00 \\
\hline No & $36(52)$ & $33(49)$ & & & \\
\hline \multicolumn{6}{|l|}{ Baseline eGFR ${ }^{b}[n(\%)]$} \\
\hline Grades $1-2$ & $29(46)$ & $34(54)$ & 4.70 & 1.21 to 18.2 & 0.022 \\
\hline Grades 3-4 & $12(80)$ & $3(20)$ & & & \\
\hline \multicolumn{6}{|l|}{ Immunotherapy agent $[n(\%)]$} \\
\hline Ipilimumab & $17(68)$ & $8(32)$ & NA & & 0.054 \\
\hline Pembrolizumab & $15(56)$ & $12(44)$ & & & \\
\hline Nivolumab & $9(35)$ & $17(65)$ & & & \\
\hline \multicolumn{6}{|c|}{ Overlap of RT and immunotherapy [ $n(\%)]$} \\
\hline Yes & $24(65)$ & $13(35)$ & 2.61 & 1.04 to 6.52 & 0.045 \\
\hline No & $17(42)$ & $24(59)$ & & & \\
\hline Mean days of overlap & $5.0 \pm 13.7$ & $1.5 \pm 2.4$ & -3.48 (mean diff.) & -9.61 to 2.66 & 0.26 \\
\hline
\end{tabular}

a Two-sided.

b As defined by The Renal Association (Bristol, U.K.): grade 1, $\geq 90 \mathrm{~mL} / \mathrm{min} / 1.73 \mathrm{~m}^{2}$; grade 2, 60-89.9 mL/min/1.73 m²; grade 3a, $45-59.9 \mathrm{~mL} /$ $\mathrm{min} / 1.73 \mathrm{~m}^{2}$; grade $3 \mathrm{~b}, 30-44.9 \mathrm{~mL} / \mathrm{min} / 1.73 \mathrm{~m}^{2}$; grade $4,15-29.9 \mathrm{~mL} / \mathrm{min} / 1.73 \mathrm{~m}^{2}$.

$\mathrm{OR}=$ odds ratio; $\mathrm{Cl}=$ confidence interval; $\mathrm{NSCLC}=$ non-small-cell lung cancer; $\mathrm{RCC}=$ renal cell carcinoma; NSAIDs = nonsteroidal anti-inflammatory drugs; eGFR - estimated glomerular filtration rate; RT = radiotherapy. 
TABLE III Logistical regression analysis of risk factors in relation to immune-related adverse events ${ }^{\mathrm{a}}$

\begin{tabular}{|c|c|c|c|c|c|c|c|c|c|}
\hline \multirow[t]{3}{*}{ Variable } & \multirow[t]{3}{*}{ Comparator } & \multirow{3}{*}{$\begin{array}{l}\text { Pts } \\
(n)\end{array}$} & \multicolumn{7}{|c|}{ Immune-related adverse events } \\
\hline & & & \multirow{2}{*}{$\begin{array}{c}\text { Yes } \\
{[n(\%)]}\end{array}$} & \multirow{2}{*}{$\begin{array}{c}\text { No } \\
{[n(\%)]}\end{array}$} & \multicolumn{2}{|c|}{ Unadjusted analysis } & \multicolumn{2}{|c|}{ Adjusted analysis } & \multirow{2}{*}{$\begin{array}{c}p \\
\text { Value }\end{array}$} \\
\hline & & & & & OR & $95 \% \mathrm{Cl}$ & OR & $95 \% \mathrm{Cl}$ & \\
\hline \multirow[t]{2}{*}{ Sex } & Men & 48 & $30(63)$ & $18(38)$ & 0.35 & 0.14 to 0.89 & 0.094 & 0.021 to 0.415 & 0.002 \\
\hline & Women & 30 & $11(37)$ & $19(63)$ & \multicolumn{2}{|c|}{ Reference } & \multicolumn{2}{|c|}{ Reference } & \\
\hline \multirow[t]{2}{*}{ History of autoimmune disease } & No & 66 & $33(50)$ & $33(50)$ & 2.00 & 0.549 to 7.29 & 9.55 & 1.34 to 68.22 & 0.025 \\
\hline & Yes & 12 & $8(67)$ & $4(33)$ & \multicolumn{2}{|c|}{ Reference } & \multicolumn{2}{|c|}{ Reference } & \\
\hline \multirow[t]{2}{*}{ Corticosteroids before immunotherapy } & No & 54 & $36(63)$ & $20(37)$ & 0.242 & 0.086 to 0.685 & 0.143 & 0.036 to 0.562 & 0.005 \\
\hline & Yes & 24 & $7(29)$ & $17(71)$ & \multicolumn{2}{|c|}{ Reference } & \multicolumn{2}{|c|}{ Reference } & \\
\hline \multirow[t]{2}{*}{ Immunotherapy agent } & PD-1 & 53 & $24(45)$ & $29(55)$ & 2.57 & 0.95 to 6.99 & 6.25 & 1.61 to 24.25 & 0.008 \\
\hline & Anti-CTLA-4 & 25 & $17(68)$ & $8(32)$ & \multicolumn{2}{|c|}{ Reference } & \multicolumn{2}{|c|}{ Reference } & \\
\hline \multirow[t]{2}{*}{ Kidney function } & Stages $1-2$ & 63 & $29(47)$ & $34(54)$ & 4.69 & 1.21 to 18.25 & 10.66 & 2.41 to 47.12 & 0.025 \\
\hline & Stages $3-4$ & 15 & $12(80)$ & $3(20)$ & \multicolumn{2}{|c|}{ Reference } & \multicolumn{2}{|c|}{ Reference } & \\
\hline
\end{tabular}

a Hosmer-Lemeshow test for goodness of fit: 0.979 .

Pts = patients; $\mathrm{OR}=$ odds ratio; $\mathrm{Cl}=$ confidence interval.

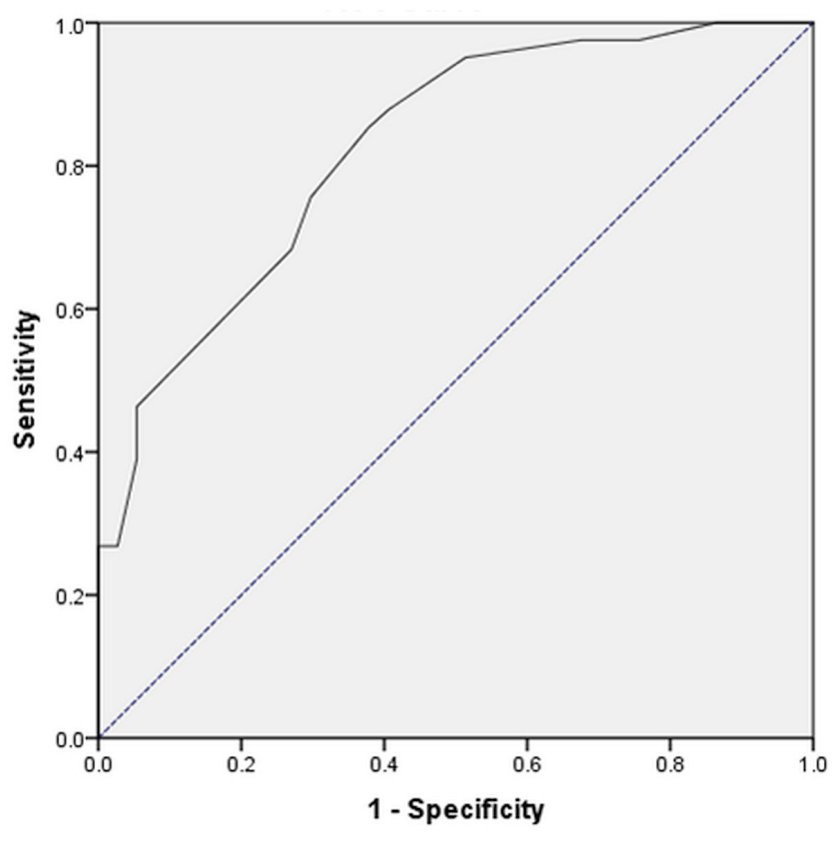

FIGURE 1 Receiver operating characteristic curve for the model, demonstrating good prediction accuracy based on the area under the curve (solid line): $82.4 \%$ (95\% confidence interval: $73.4 \%$ to $91.4 \%$ ), which was significantly different from the reference (dotted line). Diagonal segments are produced by ties.

It is well-documented that the innate and adaptive immune systems are more effective in women than in men $^{27}$, which has been ascribed to the effects of estrogen $^{28-31}$. Overall, estrogen enhances the immune response through the augmented activation of antigen-presenting cells, thereby resulting in increased antibody-mediated responses to exogenous antigens, increased T-cell cytotoxicity , and increased cytokine or chemokine levels ${ }^{27-31}$. We therefore expected women to have a higher incidence of iraEs. That expectation was inconsistent with our findings, in that female sex was associated with protection against the development of iraEs. Of the 3 most common irAE categories in the analysis, skin irAEs were associated with male sex (oR: 9.51; 95\% CI: 1.44 to $62.99 ; p=0.012$ ), but gastrointestinal irAEs (OR: $1.67 ; 95 \%$ CI: 0.31 to $9.05 ; p=$ 0.229 ) and endocrine iraEs (OR: $1.69 ; 95 \%$ CI: 0.33 to 8.72 ; $p=1.000$ ) were not found to have significant association with sex. However, those results might be underestimated because of the small sample size.

Currently, few studies have evaluated the safety profiles of immunotherapy agents in patients with underlying autoimmune diseases. In addition, such patients are typically excluded from clinical trials ${ }^{3,6,7,30-36}$. A recent review article suggested that monotherapy with CTLA- 4 or PD-1 inhibitors could be safely prescribed to patients with underlying autoimmune diseases, but the evidence for that suggestion was based on retrospective analyses. Our findings, together with the lack of available safety profile data, suggest that a decision to initiate immunotherapy should be evaluated case-by-case and weighed against the potential efficacy of immunotherapy in advanced cancer. If immunotherapy is initiated in patients with an underlying autoimmune disease, vigilant monitoring for the development of iraEs would be a crucial component of their care.

Our study suggests that CTLA-4 inhibitors are more likely than PD-1 inhibitors to induce irAEs. Although that finding is consistent with results from previous studies, the underlying mechanism remains unknown $n^{3,6,7,26,32-36}$. Given the improved efficacy of PD-1 inhibitors, single-agent CTLA-4 monoclonal antibodies are rarely used in the clinic, but they have shown some efficacy in patients who have progressed on PD-1 inhibitor therapy ${ }^{37}$.

Current recommendations suggest that PD-1 and CTLA4 inhibitors should be dosed by weight, and that no dose adjustments are necessary in relation to kidney function. Our study suggests that poor kidney function (stages 3 and 4 per the U.K. Renal Association) is correlated with a higher risk of iraE development. Monitoring the incidences of irAEs 
more prudently would therefore be important for patients with poor kidney function at baseline. Future studies evaluating the efficacy of immunotherapy in relation to kidney function would be required to determine whether renal-adjusted doses are appropriate.

\section{Limitations}

Several limitations to this study have to be addressed. First, retrospective analysis, by its nature, provides an inferior level of evidence compared with the evidence emerging from prospective studies and might be subject to confounding factors. Nonetheless, our methods minimize confounding by using the Hosmer-Lemeshow goodnessof-fit test and a receiver operating characteristic curve to adjust for confounding factors and to assess the integrity and predictive accuracy of our model.

Second, our study population consisted primarily of patients treated for melanoma, which might limit the generalizability of the findings to other cancer subtypes. Our findings are, however, representative of the general cohort of patients presenting for treatment at our academic centre. Furthermore, the understanding of cancer management is shifting toward molecular and immune checkpoint targets. We therefore believe that, compared with cancer type, cancer immunogenicity or molecular targets will play a more important role in the effects of various treatment modalities.

Lastly, our study was small in sample size. That size limitation was evident in the large confidence intervals accompanying our results. However, the study was adequately powered to detect significant differences, given the high frequency of the outcome of interest. In addition, our analysis considered PD-1 inhibitors and CTLA- 4 inhibitors alike. We are aware of the varying frequency profiles of iraEs for the different immunotherapies. Because of the small sample size, it was not feasible to conduct subgroup analyse of PD-1 inhibitors ( $n=53$ ) or CTLA- 4 inhibitors ( $n=$ 25 ) alone. Nonetheless, our study was able to confirm the higher irAE incidence rates for CTLA- 4 inhibitors compared with PD-1 inhibitors, further suggesting that our study was adequately powered despite the small sample size.

Ultimately, our study was meant to be hypothesisgenerating, and we hope that future studies involving multi-cancer centres might validate our hypothesisgenerating study.

\section{CONCLUSIONS}

Our study provides several valuable insights into an understudied topic in immunotherapy. We have highlighted several factors that can effectively predict the development of iraEs in patients receiving immunotherapy. It is of utmost importance to highlight that, of the 5 predictive factors, poor kidney function is correlated with a higher risk of irAE development, especially in view of the current guideline-recommended weight-based dosing, with no adjustment based on kidney function required. Further studies to validate the utility of our model are required.

\section{CONFLICT OF INTEREST DISCLOSURES}

We have read and understood Current Oncology's policy on disclosing conflicts of interest, and we declare that we have none.

\section{AUTHOR AFFILIATIONS}

*Department of Medicine, Queen's University, 'Hotel Dieu Hospital, ${ }^{\ddagger}$ Cancer Centre of Southeastern Ontario, and ${ }^{\S}$ Department of Endocrinology, Queen's University, Kingston, ON.

\section{REFERENCES}

1. Wilson RAM, Evans TRJ, Fraser AR, Nibbs RJB. Immune checkpoint inhibitors: new strategies to checkmate cancer. Clin Exp Immunol 2018;191:133-48.

2. Hodi FS, O'Day SJ, McDermott DF, et al. Improved survival with ipilimumab in patients with metastatic melanoma. NEngl J Med 2010;363:711-23.

3. Ribas A, HamidO, Daud A, etal. Association of pembrolizumab with tumor response and survival among patients with advanced melanoma. JAMA 2016;315:1600-9.

4. Wolchok JD, Chiarion-Sileni V, Gonzalez R, et al. Overall survival with combined nivolumab and ipilimumab in advanced melanoma. N Engl J Med 2017;377:1345-56.

5. Robert C, Schachter J, Long GV, et al. on behalf of the KEYNOTE-006 investigators. Pembrolizumab versus ipilimumab in advanced melanoma. N Engl J Med 2015;372:2521-32.

6. Borghaei H, Paz-Ares L, Horn L, et al. Nivolumab versus docetaxel in advanced nonsquamous non-small-cell lung cancer. N Engl J Med 2015;373:1627-39.

7. Motzer RJ, Escudier B, McDermott DF, et al. on behalf of the CheckMate 025 investigators. Nivolumab versus everolimus in advanced renal-cell carcinoma. NEnglJMed 2015;373:1803-13.

8. Villadolid J, Amin A. Immune checkpoint inhibitors in clinical practice: update on management of immune-related toxicities. Transl Lung Cancer Res 2015;4:560-75.

9. Champiat S, Lambotte O, Barreau E, et al. Management of immune checkpoint blockade dysimmune toxicities: a collaborative position paper. Ann Oncol 2016;27:559-74.

10. Daly LE, Power DG, O'Reilly Á, et al. The impact of body composition parameters on ipilimumab toxicity and survival in patients with metastatic melanoma. Br J Cancer 2017;116:310-17.

11. Sharma A, Thompson JA, Repaka A, Mehnert JM. Ipilimumab administration in patients with advanced melanoma and hepatitis B and C. JClin Oncol 2013;31:e370-2.

12. Minter S, Willner I, Shirai K. Ipilimumab-induced hepatitis $C$ viral suppression. J Clin Oncol 2013;31:e307-8.

13. Shelburne SA 3rd, Hamill RJ, Rodriguez-Barradas MC, et al. Immune reconstitution inflammatory syndrome: emergence of a unique syndrome during highly active antiretroviral therapy. Medicine (Baltimore) 2002;81:213-27.

14. Xiao X, Chang C. Diagnosis and classification of drug-induced autoimmunity (DIA). J Autoimmunity 2014;48-49:66-72.

15. Oh DY, Cham J, Zhang L, et al. Immune toxicities elicted by CTLA- 4 blockade in cancer patients are associated with early diversification of the T-cell repertoire. Cancer Res 2017;77:1322-30.

16. Schindler K, Harmankaya K, KukD, etal. Correlation of absolute and relative eosinophil counts with immune-related adverse events in melanoma patients treated with ipilimumab [abstract 9096]. J Clin Oncol 2014;32:. [Available online at: http:// ascopubs.org/doi/abs/10.1200/jco.2014.32.15_suppl.9096; cited 25 August 2018]

17. Shahabi V, Berman D, Chasalow SD, et al. Gene expression profiling of whole blood in ipilimumab-treated patients for identification of potential biomarkers of immune-related gastrointestinal adverse events. J Transl Med 2013;11:75.

18. Callahan MK, Yang A, Tandon S, etal. Evaluation of serum IL-17 levels during ipilimumab therapy: correlation with colitis [abstract 2505]. JClin Oncol 2011;29:. [Available online at: http:// ascopubs.org/doi/abs/10.1200/jco.2011.29.15_suppl.2505; cited 30 July 2018] 
19. Weber JS, Yang JC, Atkins MB, Disis ML. Toxicities of immunotherapy for the practitioner. J Clin Oncol 2015;33:2092-9.

20. Ilavská S, Horváthová M, Szabová M, et al. Association between the human immune response and body mass index. Human Immunol 2012;73:480-5.

21. Thajudeen B, Madhrira M, Bracamonte E, Cranmer LD. Ipilimumab granulomatous interstitial nephritis. Am J Ther 2015;22:e84-7.

22. Fadel F, El Karoui K, Knebelmann B. Anti-ctLa4 antibodyinduced lupus nephritis. N Engl J Med 2009;361:211-12.

23. Haanen JBAG, Carbonnel F, Robert C, et al. on behalf of the Esmo Guidelines Committee. Management of toxicities from immunotherapy: ESMO clinical practice guidelines for diagnosis, treatment and follow-up. Ann Oncol 2017;28(suppl 4):lv119-42.

24. United States, Department of Health and Human Services, National Institutes of Health, National Cancer Institute (NCI). Common Terminology Criteria for Adverse Events. Ver 4.0. Bethesda, MD: NCI; 2010. [Available online at: https://www. eortc.be/services/doc/ctc/CTCAE_4.03_2010-06-14_Quick Reference_5x7.pdf; cited 25 August 2018]

25. Weber JS, Hodi FS, Wolchok JD, et al. Safety profile of nivolumab monotherapy: a pooled analysis of patients with advanced melanoma. J Clin Oncol 2017;35:785-92.

26. Horvat TZ, Adel NG, Dang TO, et al. Immune-related adverse events, need for systemic immunosuppression, and effects on survival and time to treatment failure in patients with melanoma treated with ipilimumab at Memorial Sloan Kettering Cancer Centre. J Clin Oncol 2015;33:3193-8.

27. Dorak MT, Karpuzoglu E. Gender differences in cancer susceptibility: an inadequately addressed issue. Front Genet 2012;3:268

28. Pennell LM, Galligan CL, Fish EN. Sex affects immunity. J Autoimmun 2012;38:J282-91.
29. Klein SL, Hodgson A, Robinson DP. Mechanisms of sex disparities in influenza pathogenesis. J Keukoc Biol 2012;92:67-73.

30. Ghazeeri G, Abdullah L, Abbas O. Immunological differences in women compared with men: overview and contributing factors. Am J Reprod Immunol 2011;66:163-9.

31. Karpuzoglu E, Zouali M. The multi-faceted influences of estrogen on lymphocytes towards novel immune-interventions strategies for autoimmunity management. Clin Rev Allergy Immunol 2011;40:16-26.

32. Daud AI, Wolchok JD, Robert C, et al. Programmed deathligand 1 expression and response to the anti-programmed death 1 antibody pembrolizumab in melanoma.JClin Oncol 2016;34:4102-9.

33. Brahmer J, Reckamp KL, Baas $\mathrm{P}$, et al. Nivolumab versus docetaxel in advanced squamous-cell non-small-cell lung cancer. NEngl J Med 2015;373:123-35.

34. Horn L, Spigel DR, Vokes EE, et al. Nivolumab versus docetaxel in previously treated patients with advanced non-smallcell lung cancer: two-year outcomes from two randomized, open-label, phase iII trials (CheckMate 017 and CheckMate 057). J Clin Oncol 2017;35:3924-33.

35. Cella D, Grünwald V, Nathan P, et al. Quality of life in patients with advanced renal cell carcinoma given nivolumab versus everolimus in CheckMate 025: a randomised, open-label, phase 3 trial. Lancet Oncol 2016;17:994-1003.

36. Escudier B, Sharma P, McDermott DF, et al. on behalf of the CheckMate 025 investigators. CheckMate 025 randomized phase 3 study: outcomes by key baseline factors and prior therapy for nivolumab versus everolimus in advanced renal cell carcinoma. Eur Urol 2017;72:962-71.

37. Zimmer L, Apuri S, Eroglu X, et al. Ipilimumab alone or in combination with nivolumab after progression on anti-PD-1 therapy in advanced melanoma. Eur J Cancer 2017;75:47-55. 